\title{
Autosomal recessive spastic ataxia of Charlevoix-Saguenay (ARSACS)

\author{
Typical clinical and neuroimaging \\ features in a Brazilian family
}

\author{
José Luiz Pedroso', Pedro Braga-Neto', Agessandro Abrahão', \\ René Leandro Magalhães Rivero², Carolina Abdalla², \\ Nitamar Abdala², Orlando Graziani Povoas Barsottini ${ }^{1}$
}

\begin{abstract}
Autosomal recessive spastic ataxia of Charlevoix-Saguenay (ARSACS) is a neurodegenerative disorder characterized by late-infantile onset spastic ataxia and other neurological features. ARSACS has a high prevalence in northeastern Quebec, Canada. Several ARSACS cases have been reported outside Canada in recent decades. This is the first report of typical clinical and neuroimaging features in a Brazilian family with probable diagnosis of ARSACS.
\end{abstract}

Key words: autosomal recessive spastic ataxia of Charlevoix-Saguenay, early onset spastic ataxia.

Ataxia espástica autossômica recessiva de Charlevoix-Saguenay (ARSACS): aspectos clínicos e de neuroimagem típicos em uma família brasileira

\section{RESUMO}

A ataxia espástica autossômica recessiva de Charlevoix-Saguenay (ARSACS) é doença degenerativa do sistema nervoso, caracterizada por ataxia associada a espasticidade, entre outras manifestações neurológicas, de início na infância. A doença tem alta prevalência na região de Quebec, no Canadá. Muitos relatos de ARSACS têm sido descritos fora do Canadá nas últimas décadas. Nesse artigo, relatamos a primeira descrição dos aspectos clínicos e de neuroimagem típicos em uma família brasileira com provável diagnóstico de ARSACS.

Palavras-chave: ataxia espástica autossômica recessiva de Charlevoix-Saguenay, ataxia e espasticidade na infância.

Autosomal recessive spastic ataxia of Charlevoix-Saguenay (ARSACS) is an inherited neurodegenerative disorder characterized by early onset spastic ataxia, dysarthria, nystagmus, distal muscle wasting, finger and foot deformities, mixed sensorimotor neuropathy and retinal hypermyelination. It was originally described in the late 1970s, with a high prevalence among inhabitants of Charlevoix-Saguenay, northeastern Quebec, Canada ${ }^{1-3}$. ARSACS is due to mutations in a large single exon gene encoding a novel protein called sacsin that contains a heat-shock domain, which suggests that it subserves chaperone function ${ }^{4}$. In recent years several cases have been described in countries other than Canada ${ }^{2,5}$ but in spite of that this disease remains underdiagnosed outside Canada ${ }^{5}$. Therefore, we highlight the approach of patients presenting with late-infantile onset of ataxia and spasticity.

The aim of this article is to describe a Brazilian family of three siblings of non- 
consanguineous parents with clinical picture and brain magnetic resonance imaging consistent with the diagnosis of ARSACS.

\section{METHOD}

The three patients were seen at the Division of General Neurology and Cerebellar Ataxia, Department of Neurology and Neurosurgery of São Paulo Hospital, Universidade Federal de São Paulo, in São Paulo, Brazil. Their first visit was in January 2010 and they have been under diagnostic investigation since then.

All patients gave written informed consent to have their case presented in this article and approved its publication in medical literature.

\section{Cases description}

The patients were born in the city of Taubaté, state of São Paulo, southeastern Brazil. Their father is originally from the city of Itajubá, state of Minas Gerais and their mother from the city of Lorena, state of São Paulo. Their parents have not blood relatives and there is no family history of neurological disease. The children are three siblings of nonconsanguineous parents, and are all affected by the disease (Fig 1). There is no other affected family member in the family background. We did not identify any consanguineous marriages in other family members. The three patients were examined together in the first visit.

Patient 1 - Sibling 1 is a 31-year-old woman who was first evaluated in January 2010. She presented to our clinic with late-infantile onset of low progressive difficulty walking and slurred speech that started at the age of 12 months. Her brother and sister had the same symptoms. She was still able to walk in spite of the long disease duration. On neurological examination, she had cerebellar ataxia, severe spasticity in lower limbs, global hyperreflexia, dysarthria, pes cavus (Fig 2), distal amyotrophy of the limbs and nystagmus. Her cognitive function was normal. Electroneuromyography showed sensorial and motor axonal peripheral neuropathy. Ophthalmologic evaluation was normal. Magnetic resonance imaging (MRI) revealed atrophy of the cerebellum, mainly in the superior vermis, and linear hypointensities in T2 and FLAIR weighted images in the pons (Fig 3). Previous investigations in other clinics showed negative genetic test for Friedreich's ataxia, normal vitamin $\mathrm{E}$ status and negative molecular tests for spinocerebellar ataxias (SCA) type 1, 2 and 3. She was started on baclofen $30 \mathrm{mg}$ daily for spasticity and physical rehabilitation program. She was evaluated again three months later with mild improvement of spasticity.

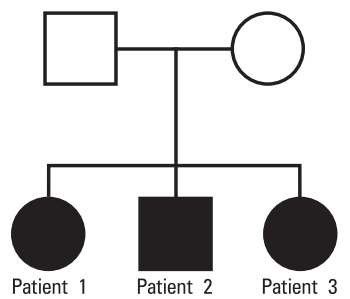

Fig 1. Heredogram showing three affected siblings of nonconsanguineous parents.

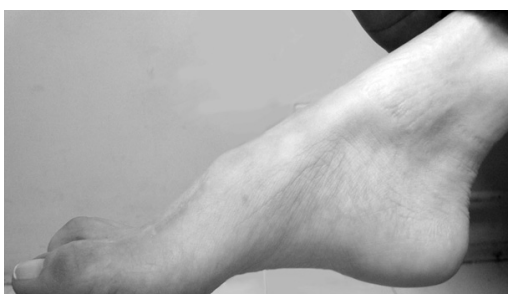

Fig 2. Pes cavus and hammer toes in $\mathrm{Pa}$ tient 1.
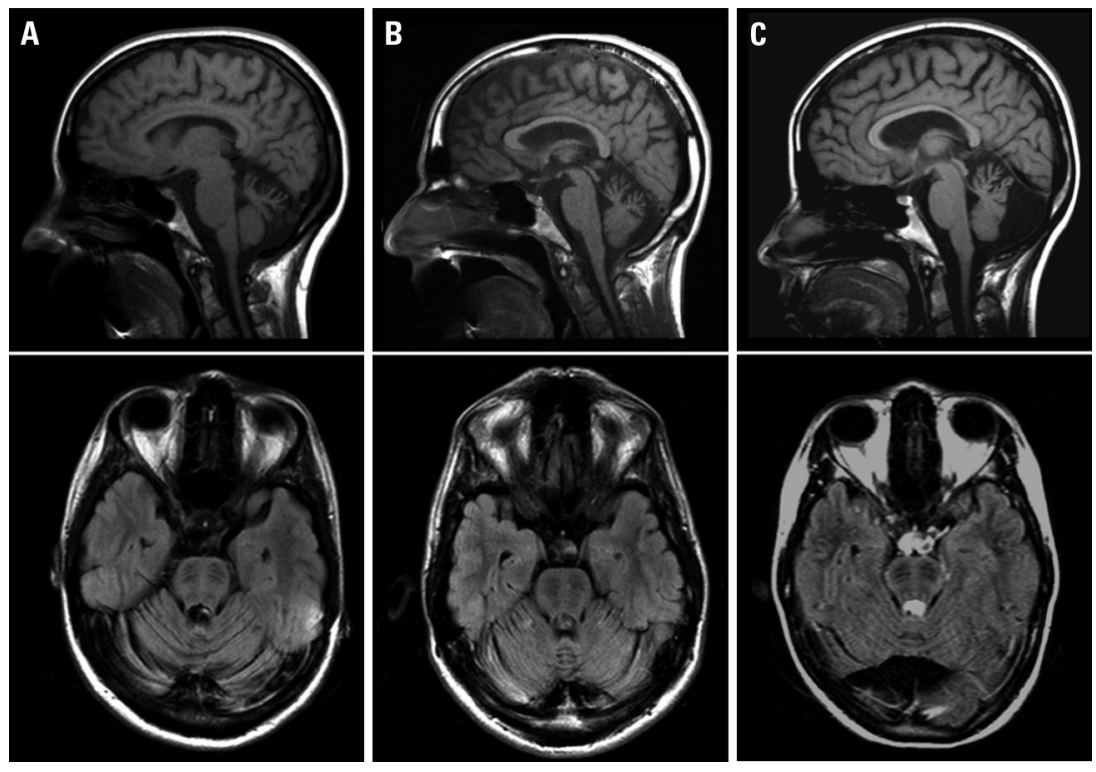

Fig 3. Sagittal T1-weighted MRI [A, B and C] showing significant atrophy of the superior vermis in all patients. There is also marked atrophy of the cervical spinal cord in the three patients. Axial FLAIR-weighted MRI [D, $E$ and $F]$ showing similar linear hypointensities in all patients. 
Patient 2 - A 30-year-old man who also came to our clinic on the same date, presenting with late-infantile onset of low progressive difficulty walking and slurred speech that also started at the age of 12 months. He had similar clinical features on examination, but less severe than Patient 1: cerebellar ataxia, spasticity, global hyperreflexia, dysarthria, pes cavus, distal amyotrophy of the limbs and nystagmus. His cognitive function was also normal. Ophthalmologic evaluation was normal. Brain MRI showed similar findings: atrophy of the cerebellum, mainly in the superior vermis, and linear hypointensities in T2 and FLAIR weighted images in the pons (Fig 3). He was started on a combination of baclofen $30 \mathrm{mg}$ daily and cyclobenzaprine $10 \mathrm{mg}$ daily, and physical rehabilitation. Three months later he reported mild symptom improvement and mild reduction in spasticity with medication.

Patient 3 - A 26-year-old woman who presented to our clinic on the same date with late-infantile onset of low progressive mild difficulty walking and slurred speech that started at the age of 12 months. On neurological examination, the same features reported in the first two cases were seen: cerebellar ataxia, spasticity, global hyperreflexia, dysarthria, pes cavus, and nystagmus. There was no amyotrophy of lower limbs and her cognitive function was also normal. Ophthalmologic evaluation was normal. Brain MRI showed similar findings as for Patients 1 and 2: cerebellar atrophy, mainly in the superior vermis, and linear hypointensities in T2 and FLAIR images in the pons (Fig 3). She opted for not taking any medication.

\section{DISCUSSION}

ARSACS was first described in 1978 as a recessive form of spastic ataxia in Quebec, Canada. Although the vast majority of ARSACS cases have been described in Canadian patients, several cases of ARSACS have been described in other countries such as Japan, Italy, Belgium, Tunisia, Spain and Turkey ${ }^{2,3,5}$. While the exact number of ARSACS patients described outside Canada is not known, approximately 30 mutations have been reported in Quebec and non-Quebec patients ${ }^{6}$. The combination of spinocerebellar ataxia with spasticity, superior vermis atrophy and pontine linear hypointensities should prompt the diagnosis of ARSACS, which may be an underdiagnosed condition outside Quebec ${ }^{7}$. ARSACS diagnosis is based on clinical manifestation, neuroimaging features and confirmed by demonstration of the deletion in the sacsin gene, located on chromosome $13 \mathrm{q}^{3}$. Neuropathologic findings showed pyramidal degeneration, cortical atrophy, atrophy of the superior cerebellar vermis and loss of motoneurons ${ }^{8}$.

The causal mutations in the SACS gene in individuals from northeastern Quebec include two founder muta- tions, a single-base deletion at position 6594 (6594delT) and a $5254 \mathrm{C}>\mathrm{T}$ nonsense mutation ${ }^{9}$. In a study of 164 alleles, 92.6\% of individuals with ARSACS born in Quebec were homozygous for the deletion 6594delT and 3.7\% were compound heterozygote for the common deletion and a missense $5254 \mathrm{C}>\mathrm{T}$ mutation ${ }^{10}$. Several novel mutations have now been described in individuals with ARSACS from Tunisia, Turkey, Italy, and Japan and previously reported in individuals who showed linkage to the $13 q 11$ locus. The phenotype observed in persons of Tunisian heritage differs from that observed in Quebec-born individuals in the very low incidence of retinal networks of myelinated fibers and the later age of onset ${ }^{11}$. Grieco et al. described three new SACS mutations in two of the original six Italian families and in two individuals the phenotype was similar to that reported in Quebec-born individuals with ARSACS except for the absence of retinal striations ${ }^{8}$. Similarly, our patients had no retinal alterations. Criscuolo et al. reported a new missense $S A C S$ mutation $(7848 \mathrm{C}>\mathrm{T})$ in a Spanish family whose phenotype was similar to the earliest presentations associated with ARSACS, thus emphasizing the widespread occurrence of ARSACS-causing mutations around the world, with the highest incidence in the Mediterranean region ${ }^{12}$.

The cardinal clinical features of ARSACS are delay in acquiring independent walk, frequent falls and gait instability. Disease progression is slow. The major manifestations in the first two decades of life are: early onset ataxia, dysarthria, nystagmus, spasticity of the legs, bilateral Babinski's reflex, and hyperreflexia. Lower limb peripheral neuropathy can also be detected later. Distal atrophy, pes cavus and hammer toes are commonly seen as the disease progresses ${ }^{2,3,13}$. In some patients, fundoscopy shows hypermyelination of fibers radiating from optic disc and embedding retinal vessels, a very peculiar finding ${ }^{3}$. Curiously, cognitive function is preserved in ARSACS, although mild mental retardation and cognitive decline have been occasionally reported. ARSACS usually appears between early childhood and preschool age (12 months old to 5.5 years old $)^{2}$. Patients become wheelchair bound by the third or fourth decade of life ${ }^{3}$. There is no effective therapy for ARSACS but physical therapy and oral drug therapy such as baclofen can help control spasticity in the early phase of the disease and may prevent tendon shortening and joint contractures.

Imaging findings (brain CT or MRI) described in ARSACS include early and progressive atrophy of the superior cerebellar vermis. Interestingly, the inferior vermis remains thicker throughout the disease. In addition, global cerebral atrophy is common in later life. However, cerebral white matter remains preserved, even in more advanced disease ${ }^{1,14}$. Cervical and thoracic spinal cord thinning are occasionally reported ${ }^{3}$. Pontine linear 
hypointensities in T2 and FLAIR images have been described as a peculiar finding in this disease, but its actual meaning remains unclear ${ }^{1,7}$.

From a practical perspective, clinical and imaging features, early nerve conduction findings, and ocular signs are all together diagnostic of ARSACS ${ }^{14}$. Most ARSACS cases first reported by Bouchard et al. ${ }^{14}$ had been diagnosed as having cerebral palsy with spastic diplegia. Confusion with cerebral palsy and secondary spastic diplegia may in part explain the apparent low incidence of ARSACS in many parts of the world ${ }^{15}$. Troyer syndrome (also known as spastic paraplegia type 20) should be considered in the differential diagnosis of ARSACS. Troyer syndrome is characterized by spastic paraplegia with distal limb amyotrophy, dysarthria, and mild cerebellar signs. It has a higher frequency in the Amish population than elsewhere in the world ${ }^{16}$. The apparent linkage of a limited number of cases from outside Quebec with the ARSACS locus on chromosome 13 otherwise suggests that other recessive progressive combined pyramidal tract and cerebellar involvement syndromes could be part of an enlarged spastic ataxia group of spinocerebellar degenerations ${ }^{14}$. Further investigation for cerebral palsy or Troyer syndrome in our three patients seems unnecessary at the moment as the clinical and neuroimaging spectrum presented here is widely compatible with ARSACS.

Our patients have major diagnostic criteria for clinical diagnosis of ARSACS: early onset (between the age of 12 and 18 months) manifested by delayed walking resulting from gait unsteadiness, progressive spastic ataxia of all limbs with paraplegia and atrophy of the superior cerebellar vermis and linear hypointensities in the pons on MRI. The combination of these features makes a probable diagnosis of ARSACS. Molecular genetic testing for the main mutation of ARSACS is not available in any center in Brazil. And testing for other SACS mutations, identified in populations outside of northeastern Quebec, are available for research purposes only.

In conclusion, this is the first documented report of clinical diagnosis of ARSACS in Brazil. We would like to stress the importance of promptly recognizing the dis- ease when late-infantile low onset progressive ataxia associated with spasticity and typical neuroimaging features are present. Additionally, cerebral palsy and Troyer syndrome should be considered in the differential diagnosis of early onset ataxia and spasticity. Imaging specialists should be familiar with typical neuroimaging features of ARSACS for more accurate diagnosis of recessive cerebellar ataxias, and neurologists should have thorough understanding about the genetics of ARSACS in view of several descriptions of the syndrome outside Canada.

\section{REFERENCES}

1. Martin MH, Bouchard JP, Sylvain M, St-Onge O, Truchon S. Autosomal recessive spastic ataxia of Charlevoix-Saguenay: a report of MR imaging in 5 patients. AJNR Am J Neuroradiol 2007;28:1606-1608.

2. Ouyang $Y$, Segers K, Bouquiaux O, et al. Novel SACS mutation in a Belgian family with sacsin-related ataxia. J Neurol Sci 2008;264:73-76.

3. Embiruçu EK, Martyn ML, Schlesinger D, Kok F. Autosomal recessive ataxias: 20 types and counting. Arq Neuropsiquiatr 2009;67:1143-1156.

4. Engert JC, Berube P, Mercier J, et al. ARSACS, a spastic ataxia common in northeastern Quebec, is caused by mutation in a new gene encoding an 11.5- Kb ORF. Nat Genet 2000;24:120-125.

5. Gomez CM. ARSACS goes global. Neurology 2004;62:10-11.

6. Takiyama Y. Autosomal recessive spastic ataxia of Charlevoix-Saguenay. Neuropathology 2006;26:368-375.

7. Van Damme P, Demaerel P, Spileers W, Robberecht W. Autosomal recessive spastic ataxia of Charlevoix-Saguenay. Neurology 2009;72:1790.

8. Grieco GS, Malandrini A, Comanducci G, et al. Novel SACS mutations in autosomal recessive spastic ataxia of Charlevoix-Saguenay type. Neurology 2004;62:103-106.

9. Mercier J, Prevost C, Engert JC, et al. Rapid detection of the sacsin mutations causing autosomal recessive spastic ataxia of Charlevoix-Saguenay. Genet Test 2001;5:255-259.

10. Richter A, Rioux JD, Bouchard JP, et al. Location score and haplotype analyses of the locus for autosomal recessive spastic ataxia of Charlevoix-Saguenay, in chromosome region 13q11. Am J Hum Genet 1999;64:768-775.

11. El Euch-Fayache G, Lalani I, Amouri R, et al. Phenotypic features and genetic findings in sacsin-related autosomal recessive ataxia in Tunisia. Arch Neurol 2003;60:982-988.

12. Criscuolo C, Sacca F, De Michele G, et al. Novel mutation of SACS gene in a Spanish family with autosomal recessive spastic ataxia. Mov Disord 2005; 20:1358-1361.

13. Garcia A, Criscuolo C, de Michele G, Berciano J. Neurophysiological study in a Spanish family with recessive spastic ataxia of Charlevoix-Saguenay. Muscle Nerve 2008;37:107-110.

14. Bouchard JP, Richter A, Mathieu J, et al. Autosomal recessive spastic ataxia of Charlevoix-Saguenay. Neuromuscul Disord 1998;8:474-479.

15. Bouchard JP, Barbeau A, Bouchard R, Bouchard RW. Autosomal recessive spastic ataxia of Charlevoix-Saguenay. Can J Neurol Sci 1978;5:61-69.

16. Patel H, Cross H, Proukakis C, et al. SPG20 is mutated in Troyer syndrome, an hereditary spastic paraplegia. Nat Genet 2002;31:347-348. 